\title{
CUIDADOS DE ENFERMAGEM OBSTÉTRICA NO PARTO NORMAL*
}

Taís Folgosa da Silva1, Guilherme Augusto Barcello Costa $^{2}$, Adriana Lenho de Figueiredo Pereira ${ }^{3}$

RESUMO: Pesquisa descritiva e retrospectiva que objetivou descrever os cuidados de enfermagem obstétrica durante o parto normal e identificar as tecnologias de cuidado no trabalho de parto e parto. Foram analisadas as informações do livro de registro dos cuidados de enfermagem aos partos normais ocorridos no ano de 2007, e identificado um total de 1287 registros de partos atendidos por enfermeiras obstétricas. As tecnologias de cuidado mais utilizadas durante o trabalho de parto foram o exercício respiratório, movimentos pélvicos, banho morno e decúbito lateral esquerdo. A posição vertical materna foi a predominante; na maioria das parturientes não foi realizada episiotomia. As enfermeiras estabelecem práticas de cuidado humanizado que possuem evidências científicas acerca do benefício, entretanto, foi identificada a influência de condutas e rotinas tradicionais de assistência ao parto, como a prescrição médica de ocitocina. PALAVRAS-CHAVE: Cuidados de enfermagem; Parto normal; Enfermagem obstétrica.

\section{OBSTETRIC NURSING CARE IN NORMAL BIRTH}

ABSTRACT: A descriptive and retrospective study which aimed to describe obstetric nursing care during normal birth and identify the techniques used. Information from the nurses' records about nursing care related to normal births was analyzed, and a total of 1287 births attended by midwives were identified. The most-used care techniques were deep breathing, pelvic movements, luke-warm baths and the left lateral position. The vertical delivery position was most common; the majority of women giving birth did not have episiotomies. The nurses established humanized practices of care with scientific evidence of benefit. However, the study also identified traditional behaviors and routines related to assistance of childbirth, such as the medical prescription of oxytocin.

KEYWORDS: Nursing care; Normal birth; Obstetric nursing.

\section{CUIDADOS DE ENFERMERÍA OBSTÉTRICA EN EL PARTO NORMAL}

RESUMEN: Investigación descriptiva y retrospectiva que objetivó describir los cuidados de enfermería obstétrica durante el parto normal e identificar las tecnologías de cuidado en el trabajo de parto y en parto. Fueron analizadas las informações del libro de registro de los cuidados de enfermería de los partos normales ocurridos en año de 2007, e identificado un total de 1287 registros de partos atendidos por enfermeras obstétricas. Las tecnologías de cuidado más utilizadas durante el trabajo de parto fueron el ejercicio respiratorio, movimientos de la pelvis, baño tibio y decúbito lateral izquierdo. La posición vertical materna fue la predominante; en la mayoría de las parturientas no fue realizada episiotomía. Las enfermeras establecen prácticas de cuidado humanizado que poseen evidencias científicas acerca del beneficio, pero fue identificada la influencia de acciones y rutinas tradicionales de asistencia al parto, como la prescrición médica de oxitocina.

PALABRAS CLAVE: Atención de enfermería; Parto normal; Enfermería obstétrica.

\footnotetext{
*Artigo elaborado a partir de monografia de conclusão do Curso de Graduação na Faculdade de Enfermagem da Universidade do Estado do Rio de Janeiro-UERJ, apresentada em 2008.

${ }^{1}$ Enfermeira Assistencial do Hospital Estadual Adão Pereira Nunes. Especialista em Enfermagem Neonatal.

${ }^{2}$ Enfermeiro da Estratégia Saúde da Família do Complexo da Maré da Secretaria Municipal de Saúde e Defesa Civil do Rio de Janeiro. Especialista em Gestão da Saúde.

${ }^{3}$ Enfermeira. Doutora em Enfermagem. Professora do Departamento de Enfermagem Materno-Infantil da UERJ.
}

\section{Autor correspondente:}

Taís Folgosa da Silva

Universidade do Estado do Rio de Janeiro

R. Torres Homem, 320 - 20551-070 - Rio de Janeiro-RJ-Brasil.

E-mail: taisfolgosa@yahoo.com.br

Recebido: $11 / 10 / 10$ Aprovado: 16/02/11 


\section{INTRODUÇÃO}

O Ministério da Saúde e a Secretaria Municipal de Saúde e Defesa Civil do Rio de Janeiro (SMSDC/RJ) têm estimulado, desde 1998, a atuação do enfermeiro obstetra na assistência ao parto normal, nas maternidades públicas. As iniciativas federais viabilizaram a emissão de Autorização de Internação Hospitalar (AIH) e a inclusão do procedimento Parto Normal sem Distócia realizado por enfermeiro obstetra na tabela do Sistema de Informações Hospitalares (SIH) do Sistema Único de Saúde e regulamentadas pelas Portarias ministeriais n. 2815/98 e 169/98 ${ }^{(1)}$.

A despeito dessas ações, o modelo de cuidado humanizado encontra resistências no cotidiano dos serviços de saúde, denotando que essas ações ainda não foram capazes de promover a superação do modelo assistencial hegemônico e produzir mudanças substanciais na cultura assistencial dos serviços de atenção obstétrica no país ${ }^{(2)}$.

Estudos demonstram que a Enfermagem tem desempenhado um papel ativo no estabelecimento de cuidados humanísticos a mulheres, auxiliando a fisiologia do parto e instituindo tecnologias de cuidado e conforto $^{(3)}$. Essas experiências de cuidado têm promovido práticas de cuidado relacionais que promovem o diálogo entre os sujeitos, usuários e profissionais, concretizando os princípios da dignidade e solidariedade, e uma postura de acolhimento ${ }^{(4)}$.

No campo da saúde, a tecnologia geralmente está associada aos aparatos biomédicos, como os equipamentos e instrumentais necessários aos procedimentos diagnósticos e terapêuticos. No entanto, seu significado é mais amplo, sendo compreendida como conhecimento aplicado que permite a prevenção, o diagnóstico e o tratamento das doenças, e a reabilitação do doente. O Ministério da Saúde considera como tecnologias em saúde os medicamentos, equipamentos e procedimentos técnicos, os sistemas organizacionais, informacionais, educacionais e de suporte e os programas e protocolos assistenciais por meio dos quais a atenção e os cuidados com a saúde são prestados à população $0^{(5)}$.

Em Enfermagem Obstétrica, as tecnologias de cuidado envolvem as técnicas, procedimentos e conhecimentos utilizados pelo enfermeiro durante o processo de cuidado, empregados nas diferentes fases do processo de parir e nascer. São tecnologias que se fundamentam na perspectiva de que a gestação, o parto e o nascimento são eventos naturais da vida humana e sua aplicação busca não intervir nos processos fisiológicos envolvidos ${ }^{(6)}$. Elas podem promover o conforto e o relaxamento, reduzir riscos e instituir cuidados eficazes, benéficos e apropriados às necessidades da clientela. Entre outras, ressaltamos as relacionadas ao uso de água, óleos essenciais, aroma e música, que proporcionem o relaxamento e conforto corporal.

Essas tecnologias, destinadas ao cuidado corporal, estimulam a movimentação corporal, como a própria deambulação, os exercícios posturais, movimentos pélvicos e o agachamento, podendo ou não empregar recursos como a bola suíça ou bobath, a cadeira de balanço obstétrica e o banquinho meia-lua.

Há tecnologias de cuidado que utilizam princípios físicos da água e da gravidade, como nos partos na água, vertical e de cócoras. Outras se fundamentam na preservação da integridade corporal e nas evidências científicas, como as técnicas de proteção perineal que evitam intervenções desnecessárias, como a episiotomia de rotina.

Estudos consideram que as tecnologias de cuidado de enfermagem estão baseadas no respeito à individualidade e autonomia do sujeito a ser cuidado, no acolhimento, na ambiência e na intersubjetividade na relação cliente-enfermeiro, agregando princípios éticos, estéticos e técnicos do cuidado ${ }^{(7-8)}$.

Considerando a importância de investigar o cuidado feito pelo enfermeiro obstétrico sob essa perspectiva, foi realizada a presente pesquisa, que objetivou descrever os cuidados de Enfermagem Obstétrica aos partos normais e identificar as tecnologias de cuidado utilizadas pelos enfermeiros no trabalho de parto e parto.

\section{METODOLOGIA}

Trata-se de um estudo descritivo e retrospectivo, no qual se utilizou a técnica da análise documental. Essa técnica de pesquisa visa o estudo de um ou vários documentos que ainda não receberam tratamento analítico, podendo ser feita no momento em que o fato ou o fenômeno ocorre, ou depois. Assim, as informações contidas no livro de registro de partos normais assistidos pelos enfermeiros obstétricos em uma maternidade pública de grande porte no Município do Rio de Janeiro foram utilizadas como fonte primária de dados para o estudo. Cabe ser esclarecido que nesse livro, há preponderantemente, a descrição dos cuidados realizados no trabalho de parto e parto, não sendo contempladas 
informações detalhadas acerca dos dados sociais e obstétricos das parturientes atendidas.

Os registros dos cuidados aos partos normais assistidos pelas enfermeiras no período de janeiro a dezembro de 2007 constituíram a amostra da pesquisa. Para delimitar o universo de partos atendidos na maternidade pesquisada, foi necessária a consulta aos dados estatísticos consolidados de nascimentos na Secretaria Municipal de Saúde e Defesa Civil (SMSDC) do Rio de Janeiro.

Foi utilizado um formulário estruturado com questões fechadas, sendo investigadas variáveis relativas aos cuidados no trabalho de parto, posição materna no parto, realização de episiotomia e condição perineal após o parto. Os dados foram armanezados em banco de dados informatizado por meio do software Microsoft Excel 2003, submetidos ao tratamento estatístico descritivo e analisados frente às evidências acerca do cuidado obstétrico tratado ou estudado na literatura nacional e internacional.

O estudo foi aprovado pelo Comitê de Ética em Pesquisa da SMSDC, obtendo o parecer n. 245A/2008.

\section{RESULTADOS}

A maternidade em questão atendeu um total de 8.200 nascidos vivos (n.v.), em 2007. Dentre estes, nasceram de parto vaginal 5.559 n.v. Foi encontrado um total de 1.287 registros de partos normais que foram cuidados por enfermeiros obstétricos, representando $44,6 \%$ da totalidade de partos vaginais atendidos na instituição.

A maioria $(743 ; 57,7 \%)$ das parturientes atendidas pelos enfermeiros era de jovens, na faixa etária entre 16 e 25 anos. Os grupos etários que tiveram menor frequência foram os extremos da idade reprodutiva, ou seja, as mulheres com idade inferior a 15 anos (49; $3,5 \%)$ e superior a 35 anos $(71 ; 5,5 \%)$.

Nos registros dos cuidados no primeiro período clínico do parto, foi encontrada elevada frequência na utilização de infusão venosa de ocitocina, correspondendo a $51,3 \%$ (661) dos atendimentos.

$\mathrm{O}$ cuidado mais registrado na assistência ao primeiro período clínico de parto foi o exercício respiratório, realizado em 77,8\% (1.002) dos atendimentos. Os demais cuidados indicados para as parturientes foram os movimentos pélvicos (509; 39,5\%), o banho morno de aspersão $(262 ; 20,4 \%)$ e o decúbito lateral esquerdo (244; 18,9\%). A deambulação, a postura de agachamento, o uso do banquinho meia-lua e da bola bobath foram os cuidados menos aplicados nessa fase, realizados em torno de 5\% das parturientes. Em 11,2\% (144) dos atendimentos, não foi registrado qualquer cuidado (Tabela 1).

Nos cuidados ao segundo período clínico do parto,

Tabela 1 - Distribuição dos cuidados prestados às parturientes, segundo os registros dos partos normais atendidos pelas enfermeiras obstétricas. Maternidade Pública. Rio de Janeiro, 2007

\begin{tabular}{lcc}
\hline Cuidados & N & \% \\
\hline Exercício respiratório & 1002 & 77,8 \\
Movimentos pélvicos & 509 & 39,5 \\
Banho morno & 262 & 20,4 \\
Decúbito lateral esquerdo & 244 & 18,9 \\
Massagem corporal & 153 & 11,9 \\
Deambulação & 64 & 5,0 \\
Postura de agachamento & 46 & 3,6 \\
Uso do banquinho meia- & 45 & 3,5 \\
lua & & \\
Uso da bola bobath & 9 & 0,7 \\
Sem registro & 144 & 11,2 \\
\hline
\end{tabular}

houve predomínio da posição vertical na expulsão, representando 78,3\% (1.008) do total de partos atendidos (Tabela 2).

A grande maioria $(1.071 ; 83,2 \%)$ das parturientes não foi submetida à episiotomia, $16,1 \%$ (207) sofreram episiotomia e em $0,7 \%$ (8) os casos não houve o registro dessa informação.

Em relação ao grupo de parturientes não sub-

Tabela 2 - Distribuição dos cuidados no parto em relação à posição materna e realização da episiotomia, segundo os registros dos partos normais atendidos pelas enfermeiras obstétricas. Maternidade Pública. Rio de Janeiro, 2007

\begin{tabular}{lcc}
\hline Posição materna & N & \% \\
\hline Vertical & 1008 & 78,3 \\
Supina & 145 & 11,2 \\
Lateral & 96 & 7,4 \\
Cócoras & 27 & 2,1 \\
Sem registro & 13 & 1,0 \\
\hline
\end{tabular}


metidas à episiotomias, a maioria $(605 ; 56,6 \%)$ teve laceração perineal de 10 grau, seguida daquelas que permaneceram com períneo íntegro (413; 38,6\%). Apenas em 4,6\% (51) das parturientes houve lacerações de 2 o grau no períneo, e não houve registro da informação em $0,2 \%$ (2) (Gráfico 1).

\section{DISCUSSÃO}

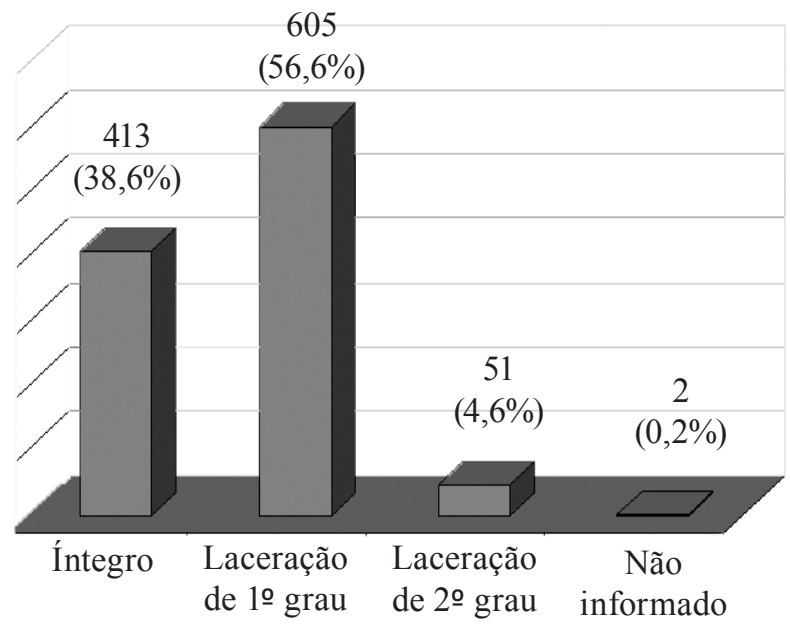

Gráfico 1 - Condição perineal após o parto das parturientes não submetidas à episiotomia, segundo os registros dos partos normais atendidos por enfermeiros obstétricos. Maternidade Pública. Rio de Janeiro, 2007

A liberdade de posição e de movimentos, assim como os métodos não farmacológicos para alívio da dor, são classificados pela Organização Mundial de Saúde, entre outras, como uma prática demonstradamente útil e que deve ser estimulada. Tais métodos enfatizam a respiração lenta e o relaxamento muscular, tendo a contribuição de um ambiente acolhedor e confortável, e da presença do acompanhante e equipe de profissionais que promovem relação de apoio e segurança $^{(9)}$. Estudos afirmam que a livre movimentação diminui a duração do primeiro estágio do trabalho de parto e que a imersão em água reduz os níveis de dor materna e a solicitação de analgesia farmacológica $^{(10-11)}$.

$\mathrm{Na}$ rotina assistencial da instituição pesquisada, a prescrição de ocitocina para indução ou aceleração do trabalho de parto é atribuição da equipe médica. $\mathrm{O}$ enfermeiro só realiza essa prescrição no pós-parto e por via intramuscular. $O$ desenho da presente pesquisa não permite estabelecer a relação entre o uso de ocitocina e o tipo de tecnologia de cuidado utilizada pelos enfermeiros. Porém, sua utilização está relacionada ao aumento da sensação dolorosa, podendo causar uma postura mais restrita ao leito, maior predisposição à ocorrência de hiperestimulação uterina e alteração na frequência cardíaca fetal ${ }^{(12)}$. Tais efeitos adversos podem requerer cuidados específicos.

Por outro lado, a baixa frequência de indicação da deambulação e o elevado percentual de infusão de ocitocina durante o trabalho de parto podem sinalizar que ainda há forte influência do modelo assistencial tradicional na maternidade pesquisada. Esse contexto, aliado a hegemonia médica no hospital, pode denotar que os enfermeiros realizam tecnologias de cuidado que promovem o conforto e o relaxamento sem gerar interferências significativas sobre a rotina assistencial, sobretudo nas condutas médicas.

A posição vertical no parto tem sido considerada benéfica devido às vantagens fisiológicas sobre a posição supina, tais como os efeitos da gravidade; a diminuição dos riscos de compressão da artéria aorta e veia cava, melhorando os indicadores sanguíneos do equilíbrio ácido-base nos recém-nascidos. Ainda, em relação à força e eficiência das contrações uterinas; a acomodação do feto durante seu trajeto pela pélvis; e a evidência radiológica de maiores diâmetros pélvicos de saída, como o anteroposterior e transverso. Por outro lado, deve ser garantida à mulher a opção de escolher a posição mais confortável no parto(13).

Em pesquisa realizada em 122 hospitais de 16 países latinoamericanos, foram estudados 416.852 nascimentos no período de 1995 a 1998. Nove entre dez mulheres primíparas, cujos filhos nasceram espontaneamente, foram submetidas à episiotomia. No Brasil, a taxa desse procedimento foi de $94,2 \%$, com proporção semelhante para hospitais públicos e priva$\operatorname{dos}^{(14)}$. Estudo realizado em uma maternidade pública da Cidade do Rio de Janeiro encontrou a frequência de episiotomia em $77,7 \%$ dos partos ${ }^{(15)}$.

A prática rotineira desse procedimento vem sendo criticada por não contar com evidências científicas dos benefícios, e as conclusões dos estudos sugerem ações para sua redução, propondo que taxas em torno de $15 \%$ devem ser alcançadas ${ }^{(16)}$. Ainda, estima-se que o país deixaria de gastar em torno de 15 a 30 milhões de dólares por ano se deixasse de realizar episiotomias desnecessárias ${ }^{(17)}$.

No Brasil, o Ministério da Saúde não preconiza uma taxa de episiotomia no parto, embora recomende a utilização restrita desse procedimento. As evidências demonstram que seu uso rotineiro não reduz o risco de trauma perineal severo (lacerações de 3 을 
e 4o graus) e não previne lesões no pólo cefálico e nem melhora os escores de Apgar. Além disso, promove maior perda sanguínea e não reduz o risco de incontinência urinária de esforço, dispareunia e dor perineal após o parto. Outro aspecto relevante é que a episiotomia é um dos únicos procedimentos realizados sem consentimento prévio da paciente ${ }^{(18)}$.

As lacerações perineais podem ser classificadas como de 10 grau (atinge a comissura labial posterior, a pele perineal e mucosa vaginal), $2 \circ$ grau (além da pele e mucosa, há ruptura da fascia e músculos do corpo perineal), 3o grau (estende-se através da pele, mucosas, corpo perineal, com a participação do esfíncter anal) e 4o grau (a ruptura estende-se até a mucosa retal, expondo o lúmen retal). Essas lesões genitais no parto vaginal podem ser prevenidas pela boa assistência, o que inclui mudança de condutas, como restrição do uso de episiotomia, de ocitocina e da posição horizontal ${ }^{(19)}$.

Outros aspectos podem estar relacionados ao trauma perineal, entre os quais a infusão intravenosa de ocitocina, o que pode levar ao desprendimento cefálico abrupto devido ao aumento das contrações e da pressão intrauterina. A aplicação de pressão no fundo uterino, durante o segundo período clínico do parto, também aumenta o risco de trauma perineal severo $^{(20)}$.

A posição materna no parto está igualmente relacionada a lesões genitais. É evidenciada a redução do risco de lacerações severas e uso de episiotomia na posição lateral; na posição vertical há maior incidência de lacerações de segundo grau e menor frequencia de episiotomia ${ }^{(19)}$.

\section{CONCLUSÃO}

A partir dos achados da pesquisa foi constatado que os enfermeiros utilizam variadas tecnologias de cuidado que promovem o relaxamento e o conforto no processo da parturição, sendo as mais empregadas o exercício respiratório, os movimentos pélvicos e o banho morno. A deambulação foi um dos cuidados menos registrados; tal achado pode estar relacionado ao fato de que mais da metade das parturientes cuidadas pelos enfermeiros recebeu infusão de ocitocina durante o trabalho de parto, sugerindo que ainda há predominância de condutas tradicionais na assistência ao parto no contexto hospitalar.

A grande maioria dos partos atendidos pelos enfermeiros foi na posição vertical, e a posição de cócoras foi a menos utilizada. Mais da metade das parturientes não teve episiotomia realizada e, neste grupo, não ocorreu casos de trauma severo ao períneo.

$\mathrm{O}$ estudo revelou que os enfermeiros estabelecem práticas de cuidado humanizado que possuem evidências científicas dos benefícios, embora as influências das condutas e rotinas assistenciais tradicionais tenham sido identificadas. As práticas assistenciais devem considerar a cidadania das mulheres e superar a compreensão dominante de que o parto é uma experiência relacionada à dor e ao medo, permitindo a qualificação da assistência ao nascimento como experiência humana dignificante e prazerosa, tendo o enfermeiro obstetra papel fundamental nesse contexto.

\section{REFERÊNCIAS}

1. Sacramento MTP, Tyrrell MAR. Vivências das enfermeiras nos cursos de especialização em enfermagem obstétrica. Rev Enferm UERJ. 2006;14(3):425-33.

2. Diniz SG. Gênero, saúde materna e o paradoxo perinatal. Rev Bras Crescimento Desenvolv Hum. 2009;19(2):313-26.

3. Progianti JM, Mouta RJO. A enfermeira obstétrica: agente estratégico na implantação de práticas do modelo humanizado em maternidades. Rev Enferm UERJ. 2009;17(2):165-9.

4. Sescato A, Souza S, Wall M. Os cuidados nãofarmacológicos para alívio da dor no trabalho de parto: orientações da equipe de enfermagem. Cogitare Enferm. 2008;13(4):585-90.

5. Ministério da Saúde (BR). Portaria n 2510, de 19 de dezembro de 2005. Institui comissão para elaboração da Política de Gestão Tecnológica no âmbito do Sistema Único de Saúde. Diário Oficial da União. [Internet] $20 \mathrm{dez} 2005$ [acesso em 08 set 2010]. Disponível: http://portal.saude.gov.br/portal/arquivos/ pdf/portaria_2510_de_19_de_dezembro_de_2005 pgts.pdf

6. Progianti JM, Vargens OMC. As enfermeiras obstétricas frente ao uso de tecnologias não invasivas de cuidado como estratégias na desmedicalização do parto. Esc Anna Nery. 2004;8(2):194-7.

7. Macedo PO, Quitete JB, Lima EC, Santos I, Vargens OMC. Tecnologias de cuidado fundamentadas pela 
teoria ambientalista de Florence Nightingale. Esc Anna Nery. 2008;12(2):341-7

8. Moreira KAP, Araújo MAM, Fernandes AFC, Braga VAB, Marques JF, Queiroz MVO. O significado do cuidado ao parto na voz de quem cuida: uma perspectiva à luz da humanização. Cogitare Enferm. 2009;14(4):720-8.

9. Organização Mundial de Saúde. Assistência ao parto normal: um guia prático. Genebra: OPAS; 1996.

10. Lawrence A, Lewis L, Hofmeyr GJ, Dowswell T, Styles C. Maternal positions and mobility during first stage labour (Cochrane Review). The Cochrane Library. Oxford: Update Software; 2009.

11. Cluett ER, Nikodem VC, McCandlish RE, Burns EE. Inmersión en agua para el embarazo, trabajo de parto y parto (Revisión Cochrane traducida). La Biblioteca Cochrane Plus; 2008.

12. Svärdby K, Nordström L, Sellström E. Primiparas with or without oxytocin augmentation: a prospective descriptive study. J Clin Nurs. 2007;16(1):179-84.

13. Gupta JK, Hofmeyr GJ. Posición de la mujer durante el período expulsivo del trabajo de parto (Revisión Cochrane traducida). La Biblioteca Cochrane Plus; 2008.

14. Althabe F, Belizán J, Bergel E. Episiotomy rates in primiparous women in Latin America: hospital based descriptive study. BMJ: British Medical Journal. 2002;324(7343):945-6.

15. D’Orsi E, Chor D, Giffin K, Angulo-Tuesta A, Peixoto G, Barbosa ASG, et al. Qualidade da atenção ao parto em maternidades do Rio de Janeiro. Rev Saúde Pública. 2005;39(4):645-54.

16. Hartmann K, Viswanathan M, Palmieri R, Gartlehner G, Thorp J Jr, Lohr KN. Outcomes of routine episiotomy: a systematic review. JAMA. 2005;293(17):2141-8.

17. Carvalho CCM, Souza ASR, Moraes Filho OB. Episiotomia seletiva: avanços baseados em evidências. Femina. 2010;38(5):265-70.

18. Rosiane MR, Aquino MMA, Mesquita MRS. A prática da episiotomia no Brasil. Rev Bras Ginecol Obstetr. 2007;29(1):1-2.

19. Costa ASC, Riesco MLG. A comparison of "hands off" versus "hands on" techniques for decreasing perineal lacerations during birth. JMWHA. 2006;51(2):106-11.

20. Hoogsteder PHJ, Pijnenborg JMA. Use of uterine fundal pressure maneuver at vaginal delivery and risk of severe perineal laceration. Arch Gynecol Obstet. 2010;281(3):579-80. 